\title{
A Fertility-Preserving Laparoscopic Management Combined With Hysteroscopy of Uterine Arteriovenous Malformation Followed by Uterine Arteries Embolization: A Case Report
}

\author{
Jing Ouyang ${ }^{1,2,3}$, Xia Wei ${ }^{1,2,3}$, Siqin Yang ${ }^{1,2,3}$, Yiru Shi ${ }^{1,2,3}$, Shiqing Hu${ }^{1,2,3}$, Jian Zhang ${ }^{1,2,3^{*}}$
}

\begin{abstract}
Objectives: The current study presented a fertility-preserving laparoscopic approach combined with hysteroscopy for the treatment of a uterine arteriovenous malformation (AVM) after a failed uterine artery embolization (UAE).

Case Presentation: The patient was diagnosed with acquired uterine AVM and underwent a UAE, followed with the recurrence of massive vaginal bleeding. The computed tomography angiography (CTA) demonstrated AVM neovascularization and the patient profoundly desired to preserve her fertility.

Results: The patient underwent a laparoscopic surgery combined with hysteroscopy during which the supply vessels of the AVM were obliterated and a 3-month GnRHa regimen was prescribed accordingly. The follow-up CTA was performed at postoperative day 3 and month 5. Both of the obtained images indicated the remission of the AVM. Thus, the patient remained asymptomatic during a 18-month follow-up after the operation.

Conclusions: In general, the laparoscopic obliteration of the supply vessels of the uterine AVM could provide an alternative to hysterectomy in patients whose embolization attempt failed and thus had a fertility-preserving desire.

Keywords: Uterine arteriovenous malformation, Laparoscopy, Hysteroscopy, Uterine arteries embolization
\end{abstract}

\section{Introduction}

Uterine arteriovenous malformation (AVM) is a vascular hamartoma of the myometrium which is characterized by the presence of shunts between the myometrial arteries and veins. In addition, uterine AVM is a potentially lifethreatening disease that can lead to acute massive vaginal bleeding. Traditional therapy for uterine AVMs centered on hysterectomy (1). However, treatments which can preserve uterus and fertility have become the preferred options by the development of medical techniques and increased requirement of uterus reserving (2-4).

The present study reports a case of a 31-year-old woman with acquired uterine AVM achieved successful management of uterine AVM using laparoscopic surgery combined with hysteroscopy. To the best of our knowledge, this is the first report of laparoscopic approach combined with hysteroscopy for the management of uterine AVM in the literature.

\section{Case Presentation}

A 31-year-old patient (gravida 1, para 0, and abortion 1) was transferred to our emergency department for acute, massive vaginal bleeding. She experienced a spontaneous miscarriage and subsequent dilatation and curettage (D/C) 3 months earlier in another hospital. The patient reported the intermittent episodes of acute vaginal bleeding after $\mathrm{D} / \mathrm{C}$ and was hospitalized in the same hospital one month earlier. The transvaginal ultrasound revealed a posteriorwall uterine AVM. Accordingly, she underwent bilateral uterine arterial angiography, which demonstrated a slightly enlarged left uterine artery and rapid arterial flow with arteriovenous shunting into a possible false aneurysm or varix (Figure 1). Then, uterine artery embolization (UAE) was performed without complication using gelatin sponge pledgets. After UAE, the patient was in a stable condition and had no further bleeding. She received a $3.75 \mathrm{mg}$ dose of triptorelin acetate and was discharged. More than one month after UAE, the patient reported the recurrence of massive vaginal bleeding and was referred to our emergency department immediately. Upon admission, she was hemodynamically stable with undetectable blood beta-human chorionic gonadotropin $(\beta$-HCG) value $(<0.1 \mathrm{IU} / \mathrm{L})$ and the hemoglobin $(\mathrm{Hb})$ level of $95 \mathrm{~g} / \mathrm{L}$. Enhanced computed tomography (CT) and three-dimensional reconstruction image based on computed tomography angiography (CTA) revealed the

Received 17 May 2018, Accepted 9 December 2018, Available online 20 April 2019

${ }^{1}$ Department of Obstetrics and Gynecology, the International Peace Maternity and Child Health Hospital, School of Medicine, Shanghai Jiaotong University, Shanghai, China. ${ }^{2}$ Shanghai Key Laboratory of Embryo Original Diseases, Shanghai, China. ${ }^{3}$ Shanghai Municipal Key Clinical Speciality, Shanghai, China.

*Corresponding Author: Jian Zhang, Tel: 86-21-64070434, E-mail: : zhangjian_ipmch@sjtu.edu.cn 


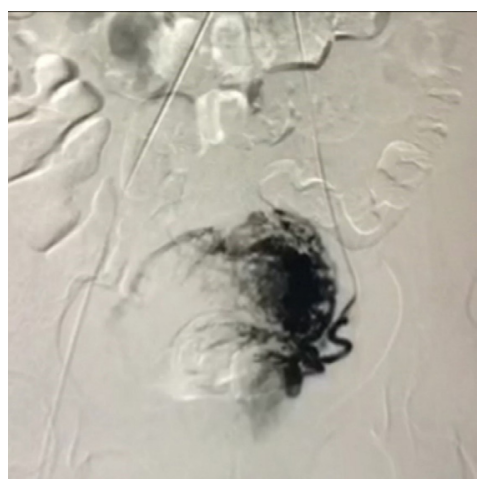

Figure 1. Embolization Procedure. Note. It demonstrates a slightly enlarged left uterine artery and rapid arterial flow with arteriovenous shunting into a possible false aneurysm or varix.

tortuous enlarged left ovarian artery and a hypervascular mass in the left posterior myometrium where tortuous veins could be observed during the arterial phase (Figure $2 \mathrm{~A}$ and $2 \mathrm{~B}$ ). Thus, uterine AVM neovascularization was considered, and the left ovarian artery was regarded as the main feeding vessel of AVM. Moreover, the patient had a profound desire to preserve her uterus. For this reason, the following surgery was considered after fully weighing the benefits and risks of different treatment options. The patient experienced intermittent vaginal bleeding during hospitalization and her $\mathrm{Hb}$ level dropped to $79.2 \mathrm{~g} / \mathrm{L}$ before the surgery as well. Therefore, two units of red blood cells were transfused to the patient.

During laparoscopy, a slight protrusion was observed on the left posterior wall of the uterus and identified as the AVM lesion. As shown in the CTA, the left ovarian artery was the main feeding vessel of AVM. In addition, the left uterine artery was replaced with a markedly enlarged tortuous parametrial vascular tangle because of the characteristics of AVMs and the previous UAE. A dilated left iliac artery was also detected due to an extensive serpentine left ovarian-uterine arterial anastomosis. These vessels of varying sizes proceeded on the surface of the uterus and within the left broad ligament. Further, the left iliac and ovarian arteries near the utero-ovarian ligament were occluded successively (double-ligated by No. 7 nonabsorbable silk suture by Johnson and Johnson, followed by coagulation with bipolar forceps). The left ovarianuterine arterial anastomosis, left utero-ovarian ligament, left round ligament, left parametrial vessels, and the ascending branches of the uterine artery were successively coagulated with bipolar forceps. Hysteroscopy showed a relatively normal appearance of endometrium on the fundus and anterior wall, along with clear bilateral uterine tubal opening. Furthermore, an approximate $40 \times 30 \mathrm{~mm}$ protrusion was found on the left posterior wall, on which the endometrium was necrotic and multiple tortuous vessels were filled with aggregated blood clots (Figure 3). The estimated blood loss was about $20 \mathrm{~mL}$ during the operation. Likewise, CTA was performed on postoperative day 3, which revealed the remission of the uterine AVM (Figure 4A and 4B). The patient was then discharged without operative complications, and GnRHa was prescribed for three months postoperatively. A followup CTA on postoperative month 5 represented continued remission of the AVM with no obvious recanalization or neovascularization (Figure 5A and 5B). The GnRHa was stopped and the cycles were regular of moderate volume. Finally, HCG levels remained undetectable and no recurrent symptoms were reported during a 18-month follow-up after the operation.

\section{Discussion}

Uterine AVM is rare and its precise morbidity is unclear. Most cases of AVMs remain undiagnosed as well (5). Uterine AVMs are classified as either congenital or
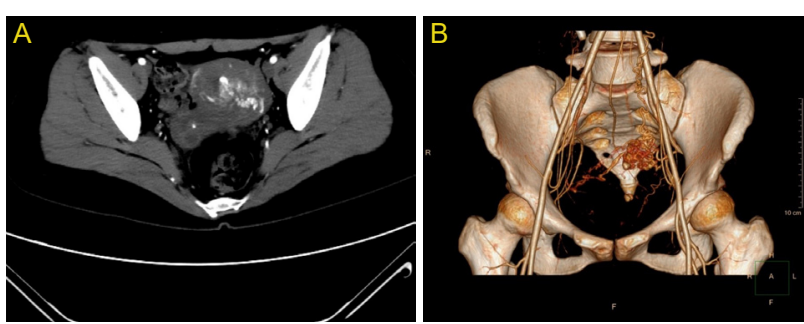

Figure 2. A) Pre-operative Computed Tomography Angiography, B) Threedimensional Reconstruction Image. Note. It displays tortuous enlarged left ovarian and a hypervascular mass in the posterior myometrium where tortuous veins could be observed during the arterial phase.

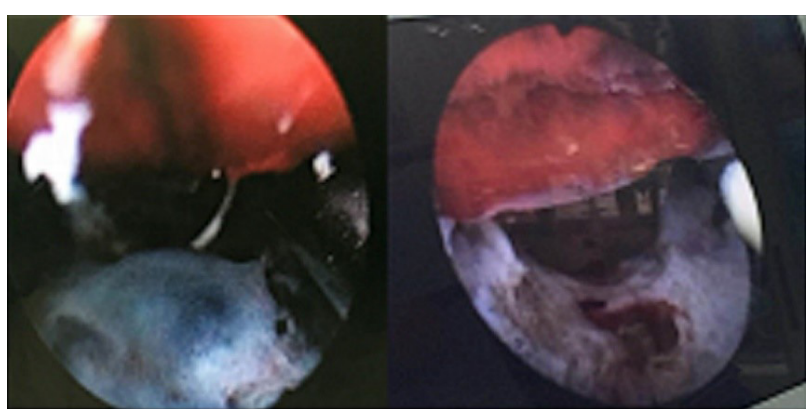

Figure 3. Hysteroscopy After Laparoscopic Vascular Ligation Procedure. Note. It shows the necrotic endometrium at the lesion site in which multiple tortuous vessels are filled with aggregated blood clots.

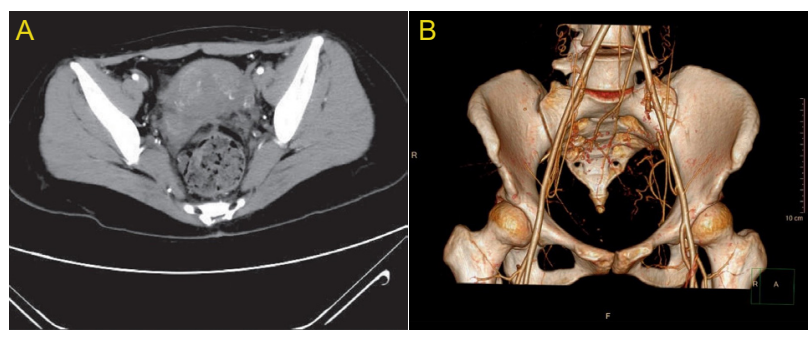

Figure 4. (A) Computed Tomography Angiography, B) 3-D Reconstruction Image. Note. It shows the remission of the arteriovenous malformation on postoperative day 3 . 

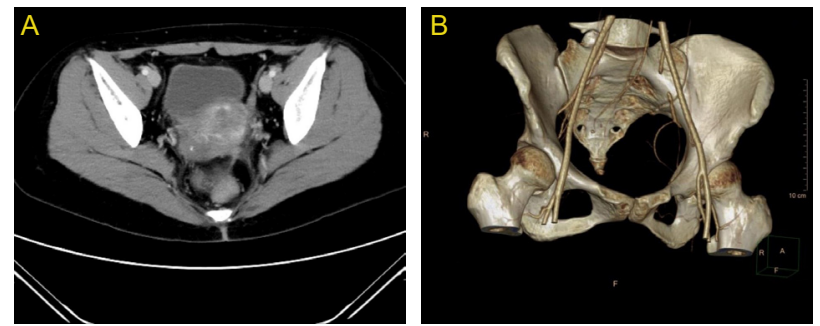

Figure 5. (A) Computed Tomography Angiography, (B) 3-D Reconstruction image. Note. It illustrates the remission of the arteriovenous malformation at 5-month follow-up.

acquired type. Congenital AVMs are extremely rare and are supposed to result from the abnormal embryologic differentiation of primordial dial vascular structures (2). However, the acquired AVMs are often associated with previous uterine trauma such as dilatation and curettage, a history of uterus surgery, and infections. Abnormal arteriovenous channels form between injured artery branches and myometrial veins. Moreover, uterine AVMs can occur as a consequence of gestational trophoblastic diseases and endometrial or cervical tumors. High levels of HCG and tumor cells could cause increased angiogenesis, which can lead to destructive changes in vascular structures and thus direct blood flow from the arteries and veins (6). No guideline is proposed for the management of uterine AVMs. Hysterectomy used to be the main treatment of uterine AVMs $(1,7)$. Clinicians develop treatments based on symptoms, age, fertility requirements, as well as the localization and size of the lesion. Expectation or medical management could be performed on hemodynamic-stable patients with small lesions and slight or no vaginal bleeding $(8,9)$. By the development of imaging and angiography techniques, endovascular embolization has become the preferred management, especially for those patients who desire to preserve their fertility. In the literature, UAE is the mostly reported procedure in terms of uterine AVM treatment. This procedure is minimally invasive while it is highly effective in controlling acute hemorrhage, and unlike hysterectomy, it allows fertility preservation. The reported success rate ranges from $79 \%$ to $93 \%$ and some patients require two or more embolization to reach AVM remission (4,10-14). Nonetheless, in some cases, AVM revascularization or neovascularization after UAE, which results in the recurrence of symptoms, can lead to the failure of UAE and thus necessitates further interventions. The corpus uteri are mainly supplied by uterine arteries while pelvic vessels are characterized by high volume and high flow with extensive collateral circulation. This can explain the relatively high rate of revascularization or neovascularization after the UAE in the treatment of uterine myoma and adenomyosis and thus leads to treatment failure or recurrent bleeding. There are a few documented cases in which repetitive endovascular embolization has successfully treated uterine AVM $(1,15)$, but surgical methods should be considered in the patient whose endovascular treatment is deemed to be contraindicated or highly risky after thorough evaluation $(1,4,11,15)$. In this case, surgical intervention is adopted given the high risks of ovarian insufficiency and potential placental abnormalities during subsequent pregnancies which are associated with the UAE. A few cases are also reported using the laparoscopic vascular ligation $(1,16,17)$. Both Hoffman et al (1) and Chen et al (16) reported the resection of the uterine AVM lesion combined with vascular ligation, which reached the successful management of the disease. However, the remission was reported $(9,18)$ and lesion retention was considered based on the possibility of spontaneous AVMs remission. Lesion retention could shorten the surgery time, reduce blood loss, leave no scars on the uterus and retain the integrity of myometrium and endometrium which could be beneficial for future conception. In this case, hysterectomy was not considered given the patient's profound desire to preserve her fertility. Then, the following surgical vascular ligation was adopted. All the arterial branches supplying the lesion were obliterated, including the left internal iliac artery, left ovarian artery, the arteries in the left round ligament, and the ascending branch of the left uterine artery. The AVM lesion was not excised and thus the integrity of myometrium and endometrium was retained to the greatest extent. This could enhance the patient's possibility of having children in the future.

Hysteroscopy can be used as a confirmatory imaging modality (19-21) and the optional management of uterine AVM (21). Calzolari et al (21) reported hysteroscopic management of uterine AVM with a $100 \%$ (11/11) success rate and a $54.55 \%(6 / 11)$ pregnancy rate post-hysteroscopy. However, only cases who were hemodynamically stable with a resolved intermenstrual bleeding episode or persistent mild or moderate bleeding were included in the report. Hysteroscopy management alone was inadequate to treat the disease for those patients with instable hemodynamics or experiencing profuse vaginal bleeding. In the present case, hysteroscopy after the laparoscopic procedure was utilized to assess the effect of supply vessel ligation and the impairment status of residual endometrium and cavity, which has great importance in the assessment of future fertility. Hysteroscopy demonstrated the successful vessel obliteration of the lesion area and left the residual endometrium almost unaffected. During the follow-up, the patients' menstrual cycles were regular and of moderate volume, and medication was stopped after post-procedure.

The researchers failed to obtain the fertility data of the patient, which is considered as the limitation of the study. The patient in the present case had no intention of pregnancy and used contraception methods since she underwent the surgery. No relevant data are available.

In conclusion, uterine AVMs are rare but could lead to life-threatening consequences. The current study demonstrated a new optional laparoscopic intervention 
combined with the hysteroscopy of the feeding vessel obliteration of uterine AVMs following a failed UAE, thus achieving the aim of preserving fertility.

\section{Conflict of Interests}

Authors declare that they have no conflict of interests.

\section{Ethical Issues}

The study was approved by the Local Ethics Committee at International Peace Maternity and Child Health Hospital in Shanghai, China (GKLW2019-01).

\section{Financial Support}

This work was supported by Shanghai Jiaotong University [grant number ZH2018QNA37].

\section{Acknowledgments}

We would like to thank LetPub webshop (http://www. letpub.com.cn) for its linguistic assistance during the preparation of the manuscript.

\section{References}

1. Hoffman MK, Meilstrup JW, Shackelford DP, Kaminski PF. Arteriovenous malformations of the uterus: an uncommon cause of vaginal bleeding. Obstet Gynecol Surv. 1997;52(12):736-740.

2. Cura M, Martinez N, Cura A, Dalsaso TJ, Elmerhi F. Arteriovenous malformations of the uterus. Acta Radiol. 2009;50(7):823-829. doi:10.1080/02841850903008792

3. Molvi SN, Dash K, Rastogi H, Khanna SB. Transcatheter embolization of uterine arteriovenous malformation: report of 2 cases and review of literature. J Minim Invasive Gynecol. 2011;18(6):812-819. doi:10.1016/j.jmig.2011.07.007

4. Ghai S, Rajan DK, Asch MR, Muradali D, Simons ME, TerBrugge KG. Efficacy of embolization in traumatic uterine vascular malformations. J Vasc Interv Radiol. 2003;14(11):1401-1408. doi:10.1097/01. rvi.0000096761.74047.7d

5. Sellers F, Palacios-Marqués A, Moliner B, Bernabeu R. Uterine arteriovenous malformation. BMJ Case Rep. 2013;2013. doi:10.1136/bcr-2012-008443

6. Kim TH, Lee HH. Presenting features of women with uterine arteriovenous malformations. Fertil Steril. 2010;94(6):2330. e2337-2310. doi:10.1016/j.fertnstert.2010.03.031

7. Patton EW, Moy I, Milad MP, Vogezang R. Fertilitypreserving management of a uterine arteriovenous malformation: a case report of uterine artery embolization (UAE) followed by laparoscopic resection. J Minim Invasive Gynecol. 2015;22(1):137-141. doi:10.1016/j. jmig.2014.07.016

8. Timmerman D, Van den Bosch T, Peeraer K, et al. Vascular malformations in the uterus: ultrasonographic diagnosis and conservative management. Eur J Obstet Gynecol Reprod Biol. 2000;92(1):171-178. doi:10.1016/s03012115(00)00443-7

9. Mekaru K, Oishi S, Akamine K, Heshiki C, Aoki Y.
Spontaneous Regression of Uterine Arteriovenous Malformations with Conservative Management. Case Rep Obstet Gynecol. 2017;2017:6437670. doi:10.1155/2017/6437670

10. McGrath S, Harding V, Lim AK, Burfitt N, Seckl MJ, Savage P. Embolization of uterine arteriovenous malformations in patients with gestational trophoblastic tumors: a review of patients at Charing Cross Hospital, 2000-2009. J Reprod Med. 2012;57(7-8):319-324.

11. Wang Z, Chen J, Shi H, et al. Efficacy and safety of embolization in iatrogenic traumatic uterine vascular malformations. Clin Radiol. 2012;67(6):541-545. doi:10.1016/j.crad.2011.11.002

12. Kwon JH, Kim GS. Obstetric iatrogenic arterial injuries of the uterus: diagnosis with US and treatment with transcatheter arterial embolization. Radiographics. 2002;22(1):35-46. doi:10.1148/radiographics.22.1.g02ja0735

13. Barral PA, Saeed-Kilani M, Tradi F, et al. Transcatheter arterial embolization with ethylene vinyl alcohol copolymer (Onyx) for the treatment of hemorrhage due to uterine arteriovenous malformations. Diagn Interv Imaging. 2017;98(5):415-421. doi:10.1016/j.diii.2016.09.003

14. Badawy SZ, Etman A, Singh M, Murphy K, Mayelli T, Philadelphia M. Uterine artery embolization: the role in obstetrics and gynecology. Clin Imaging. 2001;25(4):288295.

15. Hasegawa A, Sasaki H, Wada-Hiraike O, et al. Uterine arteriovenous fistula treated with repetitive transcatheter embolization: case report. J Minim Invasive Gynecol. 2012;19(6):780-784. doi:10.1016/j.jmig.2012.07.004

16. Chen SQ, Jiang HY, Li JB, Fan L, Liu MJ, Yao SZ. Treatment of uterine arteriovenous malformation by myometrial lesion resection combined with artery occlusion under laparoscopy: a case report and literature review. Eur J Obstet Gynecol Reprod Biol. 2013;169(2):172-176. doi:10.1016/j. ejogrb.2013.04.009

17. Levy-Zaubermann Y, Capmas P, Legendre G, Fernandez $\mathrm{H}$. Laparoscopic management of uterine arteriovenous malformation via occlusion of internal iliac arteries. J Minim Invasive Gynecol. 2012;19(6):785-788. doi:10.1016/j. jmig.2012.07.009

18. Ghizzoni V, Gabbrielli S, Mannini L, et al. Spontaneous Resolution of an Acquired Uterine Arteriovenous Malformation in an Elderly Primigravida. Am J Case Rep. 2018;19:1140-1145. doi:10.12659/ajcr.909635

19. Calzolari S, Cozzolino M, Castellacci E, Dubini V, Farruggia A, Sisti G. Hysteroscopic Management of Uterine Arteriovenous Malformation. Jsls. 2017;21(2). doi:10.4293/ jsls.2016.00109

20. Scioscia M, Zantedeschi B, Trivella G, Fratelli N, Cosma S, Minelli L. A suggestive diagnosis of uterine arteriovenous fistula based on ultrasonography and hysteroscopy. Eur J Obstet Gynecol Reprod Biol. 2012;160(1):116-117. doi:10.1016/j.ejogrb.2011.09.019

21. Calzolari S, Cozzolino M, Castellacci E. Uterine Arteriovenous Malformation: Hysteroscopic Identification Is Possible. J Minim Invasive Gynecol. 2016;23(3):293-294. doi:10.1016/j.jmig.2015.10.007

(c) 2019 The Author (s); This is an open-access article distributed under the terms of the Creative Commons Attribution License (http://creativecommons.org/licenses/by/4.0), which permits unrestricted use, distribution, and reproduction in any medium, provided the original work is properly cited. 